Zbigniew Grande*, Institute of Mathematics, Pedagogical University, Plac Weyssenhoffa 11, 85-072 Bydgoszcz, Poland. e-mail:

grande@wsp. bydgoszcz.pl

\title{
ON DISCRETE LIMITS OF SEQUENCES OF BILATERALLY QUASICONTINUOUS, BAIRE 1 FUNCTIONS
}

\begin{abstract}
In this article we show that for the discrete limit $f$ of sequence of bilaterally quasicontinuous Baire 1 functions the complement of the set of all points at which $f$ is bilaterally quasicontinuous and has Darboux property, is nowhere dense. Moreover, a construction is given of a bilaterally quasicontinuous function which is the discrete limit of a sequence of Baire 1 functions, but is not the discrete limit of any sequence of bilaterally quasicontinuous Baire 1 functions.
\end{abstract}

Let $\mathbb{R}$ be the set of all reals. In the article [3] the authors introduced the notion of the discrete convergence of sequences of functions and investigated the discrete limits in different families, for example in the family $\mathcal{C}$ of all continuous functions.

We will say that a sequence of functions $f_{n}: \mathbb{R} \rightarrow \mathbb{R}, n=1,2, \ldots$, discretely converges to the limit $f\left(f=d-\lim _{n \rightarrow \infty} f_{n}\right)$ if

$$
\forall_{x} \exists_{n(x)} \forall_{n>n(x)} f_{n}(x)=f(x) .
$$

For any family $\mathcal{P}$ denote by $B_{d}(\mathcal{P})$ the family of all discrete limits of sequences of functions from the family $\mathcal{P}$.

In [3] the class $B_{d}(\mathcal{C})$ is described and the authors observe that every strictly increasing function $f$ whose set of discontinuity points is dense does not belong to the discrete Baire system generated by $\mathcal{C}$ and the discrete convergence.

Key Words: Baire 1 class, bilateral quasicontinuity, discrete convergence, Darboux property

Mathematical Reviews subject classification: 26A15

Received by the editors January 22, 2000

*Pedagogical University grant 1999 
A function $f: \mathbb{R} \rightarrow \mathbb{R}$ is quasicontinuous (bilaterally quasicontinuous) at a point $x$ if for every positive real $\eta$ there is a nonempty open set $U \subset$ $(x-\eta, x+\eta)$ (there are open sets $V \subset(x-\eta, x)$ and $W \subset(x, x+\eta))$ such that $f(U) \subset(f(x)-\eta, f(x)+\eta)(f(V \cup W) \subset(f(x)-\eta, f(x)+\eta))([7,8])$.

In [4] it is proved that

(a) A function $f: \mathbb{R} \rightarrow \mathbb{R}$ is the discrete limit of a sequence of quasicontinuous functions if and only if the set

$$
D_{q}(f)=\{x ; f \text { is not quasicontinuous at } x\}
$$

is nowhere dense.

(b) A function $f: \mathbb{R} \rightarrow \mathbb{R}$ is the discrete limit of a sequence of bilaterally quasicontinuous functions if and only if the set

$$
D_{b q}(f)=\{x ; f \text { is not bilaterally quasicontinuous at } x\}
$$

is nowhere dense.

Let $\mathcal{D}$ denote the class of all functions $f: \mathbb{R} \rightarrow \mathbb{R}$ having Darboux property and let $Q$ (respectively $Q_{b}$ ) be the family of all quasicontinuous (bilaterally quasicontinuous) functions.

In $[6]$ the authors investigate some classes $\mathcal{P}$ of functions from $\mathbb{R}$ to $\mathbb{R}$ such that

$$
\mathcal{P} \subset B_{d}(\mathcal{D} \cap \mathcal{P}) .
$$

But both of the classes $Q$ and $Q_{b}$ do not satisfy the hypothesis of that general theorem from [6].

For this observe that

$$
Q \cap \mathcal{D} \subset Q_{b}
$$

and that for each continuous from the right and increasing function $f: \mathbb{R} \rightarrow \mathbb{R}$ discontinuous on a dense set we have

$$
D_{q}(f)=\emptyset \text { and the set } D_{b q}(f) \text { is dense. }
$$

Consequently,

$$
Q \backslash B_{d}(\mathcal{D} \cap Q)=Q \backslash B_{d}\left(\mathcal{D} \cap Q_{b}\right) \neq \emptyset .
$$

In article [5] I show two theorems describing the class $B_{d}\left(\mathcal{D} \cap Q_{b}\right)$. In our considerations we apply the following notations: Let $f: \mathbb{R} \rightarrow \mathbb{R}$ be a function and let $x \in \mathbb{R}$ be a point. Put

$$
K^{+}(f, x)=\left\{y: \exists_{\left(x_{n}\right)} x<x_{n} \rightarrow x \text { and } y=\lim _{n \rightarrow \infty} f\left(x_{n}\right)\right\},
$$




$$
K^{-}(f, x)=\left\{y: \exists_{\left(x_{n}\right)} x>x_{n} \rightarrow x \text { and } y=\lim _{n \rightarrow \infty} f\left(x_{n}\right)\right\},
$$

and recall that $x$ is a Darboux point of a function $f$ if for every positive real $r$ and for all reals $a \in\left(\min \left(f(x), \inf \left(K^{+}(f, x)\right)\right), \max \left(f(x), \sup \left(K^{+}(f, x)\right)\right)\right)$ and $b \in\left(\min \left(f(x), \inf \left(K^{-}(f, x)\right)\right), \max \left(f(x), \sup \left(K^{-}(f, x)\right)\right)\right)$ there are points $c \in(x, x+r)$ and $d \in(x-r, x)$ such that $f(c)=a$ and $f(d)=b$. It is known $([2,1])$ that a function $f$ has the Darboux property if and only if each point $x$ is a Darboux point of $f$.

Let

$$
\operatorname{Dar}(f)=\{x: x \text { is not a Darboux point of } f\} .
$$

The following two theorems are proved in [5]:

Theorem 1. Let a function $f: \mathbb{R} \rightarrow \mathbb{R}$ be such that the set $\operatorname{Dar}(f) \cup D_{b q}(f)$ is nowhere dense. Then $f$ is the discrete limit of a sequence of Darboux bilaterally quasicontinuous functions.

Theorem 2. There is a function $f: \mathbb{R} \rightarrow \mathbb{R}$ belonging to $B_{d}\left(\mathcal{D} \cap Q_{b}\right)$ such that the set Dar $(f)$ is dense.

In connection with these theorems in this article I prove the following theorem:

Theorem 3. If a function $f: \mathbb{R} \rightarrow \mathbb{R}$ is the discrete limit of a sequence of bilaterally quasicontinuous Baire 1 functions $f_{n}, n=1,2, \ldots$, then the set $D_{b q}(f) \cup \operatorname{Dar}(f)$ is nowhere dense.

Proof. By (b) the set $D_{b q}(f)$ is nowhere dense. Assume, to the contrary that there is an open interval $I$ in which the set $\operatorname{Dar}(f)$ is dense. Without loss of the generality we can suppose that $I \cap D_{b q}(f)=\emptyset$. For $n=1,2, \ldots$ let

$$
A_{n}=\left\{x \in I ; f_{k}(x)=f(x) \text { for } k \geq n\right\}
$$

Since

$$
I=\bigcup_{n=1}^{\infty} A_{n},
$$

there is a positive integer $m$ for which the set $A_{m}$ is of the second category. So, there is an open interval $J \subset I$ in which the set $A_{m}$ is dense. Since $f \mid J$ does not have the property of Darboux, there is a real

$$
s \in\left(\inf _{J} f, \sup _{J} f\right) \backslash f(J) .
$$

Let

$$
A=\{x \in J ; f(x)<s\} \text { and } B=\{x \in J ; f(x)>s\}
$$


Since the restricted function $f / J$ is bilaterally quasicontinuous, the set $E=$ $J \backslash(\operatorname{Int}(A) \cup \operatorname{Int}(B))$ is nonempty and perfect in $J$. For $n=1,2, \ldots$ let

$$
E_{n}=\left\{x \in E ;|f(x)-s| \geq \frac{1}{2^{n}}\right\} .
$$

There is a positive integer $k>m$ such that the set $E_{k} \cap A_{k}$ is of the second category in $E$. There is an open interval $L \subset J$ such that

$$
L \cap E \neq \emptyset \text { and } E \cap L \subset \operatorname{cl}\left(E_{k} \cap A_{k} \cap L\right) .
$$

Since the function $f_{k}$ is of the first class of Baire, there is a point $u \in L \cap E$ at which the restricted function $f_{k} / E$ is continuous. Consequently, there is a positive real $r$ such that

$$
(u-r, u+r) \subset L \text { and }\left|f_{m}(t)-f_{m}(u)\right|<\frac{1}{4^{k}} \text { for } t \in E \cap(u-r, u+r) .
$$

Denote by $G$ the set

$$
\left\{x \in J ; f_{j} \text { is continuous at } x \text { for } j \geq m\right\} .
$$

Evidently the set $G$ is dense in $J$ and $f=f_{j}=f_{m}$ on $G$ for $j>m$.

Observe that for $j \geq m$ and for a point $x$ which is an endpoint of some component of $\operatorname{Int}(A)$ (of $\operatorname{Int}(B)$ ) we have $f_{j}(x) \leq s\left(f_{j}(x) \geq s\right)$.

Since $E \subset \operatorname{cl}(A) \cap \operatorname{cl}(B)$ and $f_{k}$ is bilaterally quasicontinuous, we obtain

$$
f_{k}(u)=s .
$$

There is a point $w \in(u-r, u+r) \cap E_{k} \cap A_{k}$. Evidently,

$$
|f(w)-s| \geq \frac{1}{2^{k}}>\frac{1}{4^{k}}
$$

and

$$
|f(w)-s|=\left|f_{k}(w)-f_{k}(u)\right|<\frac{1}{4^{k}} .
$$

Theorem 4. There is a bilaterally quasicontinuous function $f: \mathbb{R} \rightarrow \mathbb{R}$ which is the discrete limit of a sequence of functions belonging to the first class of Baire but is not the discrete limit of any sequence of bilaterally quasicontinuous Baire 1 functions. 
Proof. Let $\left(I_{n}\right)$ be an enumeration of all open intervals with rational endpoints. We will construct our function $f$ by induction. Let $C_{1} \subset I_{1}$ be a nonempty nowhere dense perfect set and let $J_{1}=\left[\min \left(C_{1}\right), \max \left(C_{1}\right)\right]$. Then

$$
J_{1} \backslash C_{1}=\bigcup_{i=1}^{\infty}\left(a_{1, i}, b_{1, i}\right)
$$

where $\left(a_{1, i}, b_{1, i}\right)$ are components of the set $J_{1} \backslash C_{1}$.

Put

$$
f_{1}(x)=\left\{\begin{array}{ccl}
1 & \text { for } & x=a_{1, i}, i=1,2, \ldots \\
0 & \text { for } & x \in \mathbb{R} \backslash \bigcup_{i=1}^{\infty}\left[a_{1, i}, b_{1, i}\right) \\
& \text { linear on the intervals } & {\left[a_{1, i}, b_{1, i}\right], i=1,2, \ldots}
\end{array}\right.
$$

Evidently the function $f_{1}$ is bilaterally quasicontinuous.

In the second step we find a nonempty nowhere dense perfect set $C_{2} \subset$ $I_{2} \backslash C_{1}$ contained in one component of the set $I_{2} \backslash C_{1}$; denote by $J_{2}$ the closed interval $\left[\min \left(C_{2}\right), \max \left(C_{2}\right)\right]$ and by $\left(a_{2, i}, b_{2, i}\right), i=1,2, \ldots$, the components of the set $J_{2} \backslash C_{2}$. Let

$$
g_{2}(x)=\left\{\begin{array}{ccl}
\frac{1}{5} & \text { for } & x=a_{2, i}, i=1,2, \ldots \\
0 & \text { for } & x \in \mathbb{R} \backslash \bigcup_{i=1}^{\infty}\left[a_{2, i}, b_{2, i}\right) \\
& \text { linear on the intervals } & {\left[a_{2, i}, b_{2, i}\right], i=1,2, \ldots,}
\end{array}\right.
$$

and let

$$
f_{2}=f_{1}+g_{2}
$$

In general step $n(n>1)$ we find a nonempty nowhere dense perfect set $C_{n}$ contained in one component of the difference

$$
I_{n} \backslash \bigcup_{j<n} C_{j}
$$

and denote by $J_{n}$ the closed interval $\left[\min \left(C_{n}\right), \max \left(C_{n}\right)\right]$. Let $\left(a_{n, i}, b_{n, i}\right)$, $i=1,2, \ldots$, be the components of the set $J_{n} \backslash C_{n}$ and let

$$
g_{n}(x)=\left\{\begin{array}{ccl}
\frac{1}{5^{n-1}} & \text { for } & x=a_{n, i}, i=1,2, \ldots \\
0 & \text { for } & x \in \mathbb{R} \backslash \bigcup_{i=1}^{\infty}\left[a_{n, i}, b_{n, i}\right) \\
& \text { linear on the intervals } & {\left[a_{n, i}, b_{n, i}\right], i=1,2, \ldots,}
\end{array}\right.
$$

and let

$$
f_{n}=f_{n-1}+g_{n}
$$


Since for $n \geq 1$ the functions $g_{n}$ are bilaterally quasicontinuous and for $n>1$ the restricted functions $f_{n-1} / J_{n}$ are continuous, the functions $f_{n}$ are bilaterally quasicontinuous. Observe that

$$
\forall_{n \geq 1}\left|f_{n+1}-f_{n}\right| \leq \frac{1}{5^{n-1}} .
$$

So, the sequence $\left(f_{n}\right)$ uniformly converges to some function $f$, which must be bilaterally quasicontinuous.

The function $f$ is continuous at each point

$$
x \in \mathbb{R} \backslash \bigcup_{n=1}^{\infty} C_{n},
$$

continuous from the right at each point $a_{n, i}$, continuous from the left at each point $b_{n, i}, n, i \geq 1$, and

$$
f(x)=0 \text { for } x \in \bigcup_{n=1}^{\infty} C_{n} \backslash\left\{a_{n, i} ; n, i \geq 1\right\} .
$$

Observe that the function

$$
g(x)=\left\{\begin{array}{ccl}
0 & \text { for } & x \in \bigcup_{n} C_{n} \\
f(x) & \text { otherwise on } & \mathbb{R}
\end{array}\right.
$$

is of the first class of Baire as the uniform limit of the sequence of functions

$$
h_{n}(x)=\left\{\begin{array}{ccl}
0 & \text { for } & x \in \bigcup_{i \leq n} C_{i} \\
f_{n}(x) & \text { otherwise on } & \mathbb{R},
\end{array}\right.
$$

being evidently of the first Baire class.

Now, let $\left(u_{j}\right)$ be an enumeration of all points $a_{n, i}, n, i=1,2, \ldots$ and for $n=1,2, \ldots$, define

$$
\phi_{n}(x)=\left\{\begin{array}{lcl}
f(x) & \text { for } & x=u_{j}, j \leq n \\
g(x) & \text { otherwise on } & \mathbb{R} .
\end{array}\right.
$$

The functions $\phi_{n}, n \geq 1$, are of Baire 1 class and evidently

$$
f=d-\lim _{n \rightarrow \infty} \phi_{n}
$$

so $f$ is the discrete limit of a sequence of functions of Baire 1 class.

Now assume to the contrary that the function $f$ is the discrete limit of a sequence of bilaterally quasicontinuous function $\psi_{n}$ of Baire 1 class. For $n \geq 1$ let

$$
A_{n}=\left\{x ; \psi_{k}(x)=\psi_{n}(x)=f(x) \text { for } k \geq n\right\} .
$$


There is a positive integer $j$ such that the set $A_{j}$ is of the second category. Let $I$ be an open interval contained in $\operatorname{Int}\left(c l\left(A_{j}\right)\right)$. There is an integer $m>j$ with $I_{m} \subset I$. Since $f$ and $\psi_{j}$ are equal on $I_{m} \cap A_{j}$ and $\psi_{j}$ is bilaterally quasicontinuous, then

$$
f\left(a_{j, i}\right)=\psi_{j}\left(a_{j, i}\right) \text { and } f\left(b_{j, i}\right)=\psi_{j}\left(b_{j, i}\right) \text { for } i=1,2, \ldots
$$

So, the restricted function $\psi_{j} / C_{j}$ is discontinuous at each point $x \in C_{j}$ and it is not of Baire 1 class.

\section{References}

[1] A. M. Bruckner, Differentiation of real functions, Lectures Notes in Math. 659, Springer-Verlag, Berlin, 1978.

[2] A. M. Bruckner and J. Ceder, Darboux continuity, Jber. Deut. Math. Ver. 67(1965), 93-117.

[3] A. Császár and M. Laczkovich, Discrete and equal convergence, Studia Sci. Math. Hungar. 10(1975), 463-472.

[4] Z. Grande, On discrete limits of sequences of approximately continuous functions and $T_{a e}$-continuous functions, to appear.

[5] Z. Grande, On discrete limits of sequences of Darboux bilaterally quasicontinuous functions, to appear.

[6] Z. Grande and E. Strońska, Some remarks on discrete and uniform convergence, to appear.

[7] S. Kempisty, Sur les fonctions quasi-continues, Fund. Math. 19(1932), 184-197.

[8] T. Neubrunn, Quasi-continuity, Real Anal. Exch. 14(2) (1988-89), 259306. 
